PROCEEDINGS OF THE

AMERICAN MATHEMATICAL SOCIETY

Volume 134, Number 8, Pages 2393-2402

S 0002-9939(06)08241-4

Article electronically published on February 3, 2006

\title{
ON VOLUME PRODUCT INEQUALITIES FOR CONVEX SETS
}

\author{
STEFANO CAMPI AND PAOLO GRONCHI
}

(Communicated by N. Tomczak-Jaegermann)

\begin{abstract}
The volume of the polar body of a symmetric convex set $K$ of $\mathbb{R}^{d}$ is investigated. It is shown that its reciprocal is a convex function of the time $t$ along movements, in which every point of $K$ moves with constant speed parallel to a fixed direction.

This result is applied to find reverse forms of the $L^{p}$-Blaschke-Santaló inequality for two-dimensional convex sets.
\end{abstract}

\section{INTRODUCTION}

Let $K$ be a convex body in $\mathbb{R}^{d}$, that is, a $d$-dimensional compact convex set, and assume that the origin is an interior point of $K$.

In this paper, we are interested in the volume of the polar body of $K$ and, in particular, in its behavior under special continuous transformations of $K$.

The polar body $K^{*}$ of $K$ is the convex body defined by

$$
K^{*}=\left\{x \in \mathbb{R}^{d} \mid\langle x, y\rangle \leq 1, \forall y \in K\right\},
$$

where $\langle\cdot, \cdot\rangle$ is the usual scalar product in $\mathbb{R}^{d}$. Note that the polar body of $K$ strongly depends on the location of the origin. It follows from the definition that $\left(K^{*}\right)^{*}=K$.

The support function of the convex body $K$ is defined as

$$
h_{K}(u)=\max _{x \in K}\langle u, x\rangle, \forall u \in \mathbb{R}^{d},
$$

and the radial function of $K$ as

$$
\rho_{K}(u)=\max \{r \in \mathbb{R}: r u \in K\}, \forall u \in \mathbb{R}^{d} .
$$

It follows from the definitions that

$$
\rho_{K^{*}}(u)=\frac{1}{h_{K}(u)}, \forall u \in \mathbb{R}^{d} .
$$

The $d$-dimensional volume $V(K)$ of $K$ can be expressed in terms of the radial function by

$$
V(K)=\frac{1}{d} \int_{S^{d-1}} \rho_{K}^{d}(z) d z
$$

where $S^{d-1}$ is the unit sphere in $\mathbb{R}^{d}$.

Received by the editors July 27, 2004 and, in revised form, March 4, 2005.

2000 Mathematics Subject Classification. Primary 52A40.

Key words and phrases. Polar body, volume product, $L^{p}$-centroid bodies.

(C)2006 American Mathematical Society Reverts to public domain 28 years from publication 
If $K$ is an origin-symmetric convex body, then the product

$$
V(K) V\left(K^{*}\right)
$$

is called the volume product of $K$, and it is invariant under linear transformations.

For a general convex body $K$, the volume product is defined as the minimum, for $x \in K$, of $V(K) V\left((K-x)^{*}\right)$. The unique point where this minimum is attained is called the Santaló point of $K$. More precisely, the quantity $V\left((K-x)^{*}\right)^{-\frac{1}{d}}$ turns out to be a strictly concave function of $x$, as shown by A. D. Aleksandrov in A2]. In that paper, the function $V\left((K-x)^{*}\right)^{-\frac{1}{d}}$ and other means of the support function are considered in order to find estimates of solutions of the Dirichlet problem for elliptic partial differential equations (see also A1]). Volume product inequalities are also used by Talenti $[\mathrm{T}$ for obtaining sharp upper bounds of concave solutions to the Dirichlet problem for the Monge-Ampére equation in two variables. The estimates are based on the fact that, for every smooth concave function $u$ with $u\left(x_{0}\right)=1$ and vanishing on the boundary of a convex body $K$, the image $\nabla K$ of $K$ through the gradient map of $u$ contains $\left(K-x_{0}\right)^{*}$. Recall that the area of $\nabla K$ is the integral of the determinant of the Hessian matrix of $u$ extended to $K$.

One of the main questions still open in convex geometry is the problem of finding a sharp lower bound for the volume product of a convex body (see the survey article L]).

A sharp upper bound of the volume product is given by the Blaschke-Santaló inequality: For every convex body $K$ in $\mathbb{R}^{d}$

$$
V(K) V\left(K^{*}\right) \leq \kappa_{d}^{2}
$$

where $\kappa_{d}$ is the volume of the unit ball in $\mathbb{R}^{d}$. Equality holds if and only if $K$ is an ellipsoid centered at the origin (see again $[\mathrm{L}]$ ).

A sharpening of this inequality was proved by Meyer and Pajor [MP. It says that for every convex body $K$ and every affine hyperplane $H$ dividing $K$ into $K^{+}$ and $K^{-}$(both of nonzero volume) there exists a point $z$ in the relative interior of $K \cap H$ such that

$$
\frac{4 V\left(K^{+}\right) V\left(K^{-}\right) V\left((K-z)^{*}\right)}{V(K)} \leq \kappa_{d}^{2} .
$$

It was conjectured by Mahler Ma1] that the minimum of the volume product is attained when $K$ is a simplex, that is,

$$
V(K) V\left(K^{*}\right) \geq \frac{(d+1)^{d+1}}{(d !)^{2}} .
$$

In 1939 Mahler Ma2 proved (2) when $d=2$, and in 1991 Meyer Me showed that equality holds only for triangles.

For centrally symmetric convex bodies the inequality

$$
V(K) V\left(K^{*}\right) \geq \frac{4^{d}}{d !}
$$

is a conjecture as well, where the value on the right-hand side is the volume product of a $d$-parallelotope. It was proved in dimension two by Mahler Ma2. Reisner Re2 showed that parallelograms are the only minimizers, for $d=2$. Saint Raymond [SR] showed in dimension $d>3$ that there are convex bodies, other than parallelotopes and their polars, for which (3) is an equality. He also proved, for every $d$, that (3) holds true for all the affine images of convex sets symmetric with respect to the coordinate hyperplanes. Inequality (3) was proved by Reisner [Re1, Re2 for all 
zonoids. Such a class can be defined as the closure, in the Hausdorff metric, of finite vector sums of segments. A simpler proof of Reisner's result was given in GMR.

Bourgain and Milman [BM] proved that there exists a constant $c$, not depending on the dimension, such that

$$
V(K) V\left(K^{*}\right) \geq c^{d} \kappa_{d}^{2} .
$$

In the present paper we shall consider movements of a given origin-symmetric convex body $K$, obtained by assigning to each point of $K$ a speed, independent of the time $t$, which is parallel to a fixed direction (see Section 2). Denoting by $K_{t}$ the convex hull of the resulting points at time $t$, we shall prove in Section 3 that $V^{-1}\left(K_{t}^{*}\right)$ is a convex function of $t$ (Theorem 1 ), where $K_{t}^{*}=\left(K_{t}\right)^{*}$.

The main tool in the proof is the so-called Borell-Brascamp-Lieb inequality, which deals with the $p$-means of functions and their integrals. Such an inequality is an extension of the Prékopa-Leindler inequality and can be considered as an inverse Hölder inequality. The importance of the Borell-Brascamp-Lieb inequality and its links with other famous inequalities (e.g., the isoperimetric inequality) is widely described in the survey article by Gardner G2.

Using Theorem 1, one recovers for origin-symmetric convex bodies the BlaschkeSantaló inequality, as well as Mahler's inequality (3) in the two-dimensional case.

In Section 4 we apply Theorem 1 to the study of the functional

$$
V\left(\Gamma_{p}^{*} K\right) V(K),
$$

where $\Gamma_{p} K$ is the $L^{p}$-centroid body of $K$. We obtain an alternative proof of a result by Lutwak and Zhang [LZ], stating that, in the class of all convex bodies of $\mathbb{R}^{d}$, the quantity (4) attains its maximum when $K$ is an ellipsoid centered at the origin. Such a result is called the $L^{p}$-Blaschke-Santaló inequality. This name comes from the fact that when $K$ is origin-symmetric and $p$ goes to infinity, (4) tends to the volume product.

We also address the question of a reverse $L^{p}$-Blaschke-Santaló inequality and use Theorem 1 to establish such an inequality in dimension $d=2$. Namely, we are able to show that, among all convex figures containing the origin, (4) is minimized when $K$ is a triangle with a vertex at the origin (Theorem 3). Triangles are still minimizers of (4) in the class of all convex bodies with their barycenter at the origin (Theorem 4). Finally, if one takes the maximum value of (4) with respect to all possible locations of $K$ in the plane, then such a value is minimum for triangles (Theorem 5).

We also establish a reverse $L^{p}$-Blaschke-Santaló inequality for centrally symmetric convex figures and show that the extremal figures are parallelograms instead of triangles.

\section{Shadow Systems}

A shadow system along the direction $v \in S^{d-1}$ is a family of convex sets $K_{t} \subset \mathbb{R}^{d}$ such that

$$
K_{t}=\operatorname{conv}\left\{x+\alpha(x) t v: x \in A \subset \mathbb{R}^{d}\right\},
$$

where conv denotes convex hull, $A$ is an arbitrary bounded set of points, $\alpha$ is a bounded function on $A$, and $t$ belongs to an interval of the real axis. The notion of shadow system was introduced by Rogers and Shephard ([RS and $[\mathrm{Sh}]$ ), who proved the basic fact that the volume of $K_{t}$ is a convex function of $t$. This result is a powerful tool for obtaining geometric inequalities of isoperimetric type. 
As suggested by Shephard in $\underline{\mathbf{S h}}$, a shadow system can be seen as the family of projections of a $(d+1)$-dimensional convex set. Namely, let $e_{1}, e_{2}, \ldots, e_{d+1}$ be an orthonormal basis of $\mathbb{R}^{d+1}$, and let $\tilde{K}$ be the $(d+1)$-dimensional convex set defined by

$$
\tilde{K}=\operatorname{conv}\left\{x+\alpha(x) e_{d+1}: x \in A \subset e_{d+1}^{\perp}\right\},
$$

where $e_{d+1}^{\perp}=\left\{w \in \mathbb{R}^{d+1}:\left\langle w, e_{d+1}\right\rangle=0\right\}$.

For each $t$, the projections of $\tilde{K}$ onto a hyperplane orthogonal to $e_{d+1}$ along the directions $e_{d+1}-t v$ are just the family $K_{t}$. This interpretation permits one to find that not only the volume but also other geometric quantities are convex functions of the parameter $t$ of a shadow system. Indeed, projecting all the sets of a shadow system onto a linear space along a fixed direction produces another shadow system.

So, for example, the brightness function of $K_{t}$ is a convex function of $t$. Recall that the brightness of a convex set, as a function of $u \in S^{d-1}$ is the $(d-1)$ dimensional volume of its orthogonal projection onto $u^{\perp}$. Besides, by the Cauchy formula, the surface area of a convex set is the average of its brightness function (see, for example [Sc], p. 295). Therefore, the surface area of $K_{t}$ is still a convex function with respect to $t$. Analogously, by taking the projections of $K_{t}$ onto lowerdimensional linear spaces, it turns out that all the so-called intrinsic volumes of $K_{t}$ (see $[\mathrm{Sc}$, p. 210) are convex functions with respect to the parameter $t$.

Steiner symmetrization arises from a particular shadow system. Indeed, if one moves each chord of $K$ parallel to $v$ so that, at $t=1$, such a chord is in the reflected position with respect to $v^{\perp}$, then, at $t=\frac{1}{2}$, the union of all the chords is nothing but the Steiner symmetral of $K$ with respect to $v$. Precisely, let $K$ be represented by

$$
K=\left\{x+y v \in \mathbb{R}^{d}: x \in K \mid v^{\perp}, y \in \mathbb{R}, f(x) \leq y \leq g(x)\right\},
$$

where $\cdot \mid v^{\perp}$ denotes the orthogonal projection onto $v^{\perp}$ and $f$ and $-g$ are convex functions on $K \mid v^{\perp}$. The shadow system along $v$, with speed $\alpha(x)=-f\left(x \mid v^{\perp}\right)-$ $g\left(x \mid v^{\perp}\right)$, is such that $K_{1 / 2}$ coincides with the Steiner symmetral of $K$ about $v^{\perp}$.

A shadow system with a speed function constant on each chord parallel to the direction of the movement is called a parallel chord movement.

A way of exploiting convexity is the one suggested by the following argument of Shephard Sh]: If a functional defined in the class of all convex sets is continuous, invariant under reflections and convex with respect to the parameter $t$ of any shadow system, then it attains its minimum at the ball among all sets of given volume. Here the continuity refers to the Hausdorff metric.

This statement follows by the use of Steiner symmetrization. It is worth recalling that such a symmetrization keeps the volume unchanged and leads, if suitably repeated, to a ball.

Consequences of this procedure are classical isoperimetric-type inequalities for intrinsic volumes (see [BZ, p. 144, G1, p. 372). Moreover, other geometric functionals have the same convexity property under shadow systems (see, for example, [Sh], CCG], CG1]).

\section{Polar Bodies And Shadow Systems: The Main Result}

In this section we describe the behavior of the volume of the polar body along the movement associated to an origin-symmetric shadow system. 
Definition 1. Let $p \neq 0$. A nonnegative function $f$ on $\mathbb{R}^{d}$ is called $p$-concave on a convex set $A$ if

$$
f((1-\lambda) x+\lambda y) \geq\left[(1-\lambda) f(x)^{p}+\lambda f(y)^{p}\right]^{1 / p}
$$

for all $x, y \in A$ and $0<\lambda<1$.

Note that if $p<0$, then $f$ is $p$-concave if and only if $f^{p}$ is convex. The definition above can be extended to the case $p=0$ by continuity (see [G2]).

In what follows we will use the following result to deduce the behavior of integrals of a family of $p$-concave functions.

Borell-Brascamp-Lieb inequality. If $0<\lambda<1,-1 / d \leq p \leq \infty$, and $f, g, h$ are nonnegative integrable functions on $\mathbb{R}^{d}$ satisfying

$$
h((1-\lambda) x+\lambda y) \geq\left[(1-\lambda) f(x)^{p}+\lambda g(y)^{p}\right]^{1 / p},
$$

for all $x, y \in \mathbb{R}^{d}$, then

$$
\int_{\mathbb{R}^{d}} h(x) d x \geq\left[(1-\lambda)\left(\int_{\mathbb{R}^{d}} f(x) d x\right)^{p /(d p+1)}+\lambda\left(\int_{\mathbb{R}^{d}} g(x) d x\right)^{p /(d p+1)}\right]^{(d p+1) / p} .
$$

See G2 for an extensive list of references on this inequality.

Corollary. Let $F(x, y)$ be a nonnegative $p$-concave function on $\mathbb{R}^{n} \times \mathbb{R}^{m}, p \geq-1 / n$. If, for every $y$ in $\mathbb{R}^{m}$, the integral

$$
\int_{\mathbb{R}^{n}} F(x, y) d x
$$

exists, then it is a $\frac{p}{n p+1}$-concave function of $y$.

Proof. Take $y_{0}, y_{1} \in \mathbb{R}^{m}$ and fix $\lambda \in(0,1)$. Let $y_{\lambda}=(1-\lambda) y_{0}+\lambda y_{1}$, and

$$
f(x)=F\left(x, y_{0}\right), \quad g(x)=F\left(x, y_{1}\right), h(x)=F\left(x, y_{\lambda}\right) .
$$

For every $x_{0}, x_{1} \in \mathbb{R}^{n}$, we have that

$$
h^{p}\left((1-\lambda) x_{0}+\lambda x_{1}\right)=F^{p}\left((1-\lambda) x_{0}+\lambda x_{1}, y_{\lambda}\right) \geq(1-\lambda) f^{p}\left(x_{0}\right)+\lambda g^{p}\left(x_{1}\right),
$$

where we used the $p$-concavity of $F$.

The Borell-Brascamp-Lieb inequality now gives the desired conclusion.

We are now ready to state and prove the following theorem.

Theorem 1. If $K_{t}, t \in[0,1]$, is a shadow system of origin-symmetric convex bodies in $\mathbb{R}^{d}$, then $V\left(K_{t}^{*}\right)^{-1}$ is a convex function of $t$.

Proof. Let $K_{t}$ be a shadow system as stated above. Therefore, there exists a bounded function $\alpha$ on $K_{0}$ such that

$$
K_{t}=\operatorname{conv}\left\{x+\alpha(x) t v: x \in K_{0}\right\} .
$$

Let us introduce the $(d+1)$-dimensional convex body

$$
\tilde{K}=\operatorname{conv}\left\{x+\alpha(x) e_{d+1}: x \in K_{0}\right\},
$$

so that $K_{t}$ can be thought of as the projection along the direction $e_{d+1}-t v$ of $\tilde{K}$ onto $e_{d+1}^{\perp}$. 
There is a connection between the support functions $h_{K_{t}}, t \in[0,1]$, and the support function of $\tilde{K}$. Precisely, for $u \in e_{d+1}^{\perp}$,

$$
h_{K_{t}}(u)=\max _{x \in K_{t}}\langle u, x\rangle=\max _{x \in K_{0}}\langle u, x+\alpha(x) t v\rangle .
$$

Note that $\langle u, x+\alpha(x) t v\rangle=\left\langle u+t\langle u, v\rangle e_{d+1}, x+\alpha(x) e_{d+1}\right\rangle$; moreover, as $x$ runs in $K_{0}, x+\alpha(x) e_{d+1}$ covers all the extreme points of $\tilde{K}$. Recall that an extreme point of a convex set is a point which cannot be expressed as a convex linear combination of two different points of the set. Hence,

$$
h_{K_{t}}(u)=\max _{y \in \tilde{K}}\left\langle u+t\langle u, v\rangle e_{d+1}, y\right\rangle=h_{\tilde{K}}\left(u+t\langle u, v\rangle e_{d+1}\right) .
$$

We know that

$$
V\left(K_{t}^{*}\right)=\frac{1}{d} \int_{S^{d-1}} h_{K_{t}}^{-d}(z) d z .
$$

Let $D^{d-1}=\left\{x \in v^{\perp}:\|x\| \leq 1\right\}$; thus $S_{+}^{d-1}=\left\{z \in S^{d-1}:\langle z, v\rangle \geq 0\right\}$ is the graph of the function $\sqrt{1-\|x\|^{2}}, x \in D^{d-1}$. Consequently,

$$
\int_{S^{d-1}} h_{K_{t}}^{-d}(z) d z=2 \int_{D^{d-1}} \frac{h_{K_{t}}^{-d}\left(x+\sqrt{1-\|x\|^{2}} v\right)}{\sqrt{1-\|x\|^{2}}} d x,
$$

where we took into account that $K_{t}$ is origin-symmetric.

By (5),

$$
h_{K_{t}}\left(x+\sqrt{1-\|x\|^{2}} v\right)=h_{\tilde{K}}\left(x+\sqrt{1-\|x\|^{2}} v+t \sqrt{1-\|x\|^{2}} e_{d+1}\right) .
$$

Therefore, from $(6),(7)$ and (8) we obtain that

$$
V\left(K_{t}^{*}\right)=\frac{2}{d} \int_{D^{d-1}} \frac{h_{\tilde{K}}^{-d}\left(\frac{x}{\sqrt{1-\|x\|^{2}}}+v+t e_{d+1}\right)}{\left(1-\|x\|^{2}\right)^{\frac{d+1}{2}}} d x,
$$

where we also used the homogeneity of the support function.

Use the change of variables $y=\frac{x}{\sqrt{1-\|x\|^{2}}}$ in the latter integral. It is easy to check that the determinant of the Jacobian matrix of that map is just $\left(1-\|x\|^{2}\right)^{-\frac{d+1}{2}}$. We conclude that

$$
V\left(K_{t}^{*}\right)=\frac{2}{d} \int_{\mathbb{R}^{d-1}} h_{\tilde{K}}^{-d}\left(y+v+t e_{d+1}\right) d y .
$$

The function $h_{\tilde{K}}$ is convex in $\mathbb{R}^{d+1}$; therefore, by the corollary of the BorellBrascamp-Lieb inequality, $V\left(K_{t}^{*}\right)$ is $p$-concave with $p=\frac{-1 / d}{1-(d-1) / d}=-1$.

\section{Consequences}

By Theorem 1, if we apply Shephard's argument, quoted in the previous section, to the reciprocal of the volume product, we immediately deduce the BlaschkeSantaló inequality (1), for origin-symmetric convex bodies.

A different argument, that we shall use explicitly in the proof of Theorem 3, leads to the Mahler's inequality (3), for plane convex figures.

Both the above results can be seen as special instances of a more general class of inequalities, which involve the $L^{p}$-centroid body of a body $K$. 
For each real number $p \geq 1$, the $L^{p}$-centroid body $\Gamma_{p} K$ of $K$ is the convex body with support function

$$
h_{\Gamma_{p} K}(u)=\left\{\frac{1}{c_{d, p} V(K)} \int_{K}|\langle u, z\rangle|^{p} d z\right\}^{\frac{1}{p}}, u \in \mathbb{R}^{d},
$$

where

$$
c_{d, p}=\frac{\kappa_{d+p}}{\kappa_{2} \kappa_{d} \kappa_{p-1}}
$$

and

$$
\kappa_{r}=\pi^{\frac{r}{2}} / \Gamma\left(1+\frac{r}{2}\right) .
$$

Note that $\kappa_{d}$ is the volume of the unit ball $B^{d}$ of $\mathbb{R}^{d}$, and the constant $c_{d, p}$ is such that $\Gamma_{p} B^{d}=B^{d}$, for every $d$ and $p$.

This definition is due to Lutwak and Zhang [LZ] and extends to $p>1$ the concept of centroid body, corresponding to $p=1$. For $p=2$, formula (9) gives, up to a constant, the Legendre ellipsoid of $K$. If one defines $\Gamma_{\infty} K$ as the limit of $\Gamma_{p} K$ when $p \rightarrow \infty$, then $\Gamma_{\infty} K=\operatorname{conv}(K \cup(-K))$.

Inequalities involving the volume of $\Gamma_{p} K$ can be found in [LZ, [LYZ, CG1] and CG2. Here we are interested in the functional (4), with $p \geq 1$, and we set for simplicity

$$
G_{p}(K)=V\left(\Gamma_{p}^{*} K\right) V(K) .
$$

It is easy to check that $G_{p}$ is continuous and invariant under reflection about hyperplanes through the origin. Moreover, for every linear map $L, \Gamma_{p}(L K)=L \Gamma_{p} K$ (see, e.g., CG2]).

In $[\mathrm{LZ}]$ it is shown that the maximum of $G_{p}(K)$ is attained if and only if $K$ is an origin-symmetric ellipsoid. For $p=\infty$, this yields the Blaschke-Santaló inequality (1) for centrally symmetric convex bodies.

The "if" part of the Lutwak-Zhang result can now also be deduced from Shephard's argument and the following theorem.

Theorem 2. If $K_{t}, t \in[0,1]$ is a parallel chord movement, then $V\left(\Gamma_{p}^{*} K_{t}\right)^{-1}$ is a convex function of $t$.

Theorem 2 is a consequence of Theorem 1 and the following one, proved in CG1.

If $K_{t}, t \in[0,1]$ is a parallel chord movement along the direction $v$, then $\Gamma_{p} K_{t}$ is a shadow system along the same direction $v$.

Let us now deal with the problem of finding lower bounds for $G_{p}$. Clearly, the functional $G_{p}(K)$ tends to zero as $K$ moves away from the origin. So, natural ways for posing the problem is to restrict ourselves to convex bodies containing the origin or to bodies with their barycenter at the origin or else to consider the functional

$$
M_{p}(K)=\max _{x \in \mathbb{R}^{d}} G_{p}(K-x) .
$$

Note that $G_{p}(K-x)^{-1}$ is a convex function of $x$, by Theorem 2 .

In all these three cases, the Lutwak-Zhang inequality implies that the maximum is attained only at ellipsoids centered at the origin.

The search of minimizers, for $d=2$, is guided by Theorem 2, through a method already used by the authors in CG2.

Theorem 3. Let $d=2$. The minimum of $G_{p}(K)$ in the class of all convex bodies containing the origin is attained if $K$ is a triangle with one vertex at the origin. 
The minimum of $G_{p}(K)$ in the class of all centrally symmetric convex bodies containing the origin is attained if $K$ is a parallelogram with one vertex at the origin.

Proof. Let $P$ be a polygon with $n$ vertices, $n>3$. Since we are interested in minimizers of $G_{p}$, we can assume that the origin is at one of the vertices of $P$. Take three consecutive vertices $v_{1}, v_{2}, v_{3}$, different from the origin. Denote by $u$ a direction of the line through $v_{1}$ and $v_{3}$, and consider the following shadow system. Assign speed $u$ to $v_{2}$ and 0 to the other vertices and consider the resulting shadow system $P_{t}$, where $t \in\left[t_{0}, t_{1}\right]$, which is the largest interval such that the area of $P_{t}$ is constant for all $t \in\left[t_{0}, t_{1}\right]$. Note that $P_{t}, t \in\left[t_{0}, t_{1}\right]$, is a parallel chord movement such that only the triangle $v_{1} v_{2} v_{3}$ moves and then the origin remains in $P_{t}$, for all $t$. Moreover, $P_{0}=P$, and $P_{t_{0}}$ and $P_{t_{1}}$ have exactly $n-1$ vertices.

By Theorem 2,

$$
G_{p}(P) \geq \min \left\{G_{p}\left(P_{t_{0}}\right), G_{p}\left(P_{t_{1}}\right)\right\} .
$$

If $n>4$, iterations of this argument lead to the conclusion that

$$
G_{p}(P) \geq G_{p}(T),
$$

where $T$ is a triangle with one vertex at the origin. The linear invariance of $G_{p}$ ensures that $G_{p}(T)$ attains the same value whichever triangle, with a vertex at the origin, we consider. By the continuity of $G_{p}$, an approximation argument ends the proof of the first statement.

Let $P$ be a centrally symmetric polygon with $2 n$ vertices, $n>2$, and assume that the center of $P$ is $c$ and that the origin is at one of the vertices of $P$. Take three consecutive vertices $v_{1}, v_{2}, v_{3}$, with $v_{2} \neq 0$. If we move the triangle $v_{1} v_{2} v_{3}$ as in the nonsymmetric case and the triangle with vertices $2 c-v_{1}, 2 c-v_{2}, 2 c-v_{3}$ symmetrically with respect to $c$, we define a shadow system $P_{t}$ which leads to a centrally symmetric polygon with $2 n-2$ vertices such that the value of $G_{p}$ is not increased. Possible iterations end the proof.

Note that for plane centrally symmetric convex bodies, the Mahler inequality (3) can also be deduced from Theorem 3 by taking $p=\infty$.

Theorem 4. Let $d=2$. The minimum of $G_{p}(K)$ in the class of all convex bodies with their barycenter at the origin is attained if $K$ is a triangle.

The minimum of $G_{p}(K)$ in the class of all origin-symmetric convex bodies is attained if $K$ is a parallelogram.

Proof. To prove both statements we can follow the same outlines of the previous proof. What we need here to add is that the barycenter of $P_{t}$ must remain at the origin for every $t$. The key property is that, along a parallel chord movement $K_{t}$, the barycenter $c_{K_{t}}$ of $K_{t}$ moves with constant speed parallel to the direction $u$ of the movement. Indeed,

$$
\begin{aligned}
c_{K_{t}} & =\frac{1}{V\left(K_{t}\right)} \int_{K_{t}} x d x=\frac{1}{V\left(K_{t_{0}}\right)} \int_{K_{t_{0}}}(x+\alpha(x) t u) d x \\
& =c_{K_{t_{0}}}+\frac{t u}{V\left(K_{t_{0}}\right)} \int_{K_{t_{0}}} \alpha(x) d x .
\end{aligned}
$$

Therefore, the family $P_{t}-c_{P_{t}}, t \in\left[t_{0}, t_{1}\right]$, is a parallel chord movement of bodies with their barycenter at the origin. 
Theorem 5. Let $d=2$. The minimum of $M_{p}(K)$ in the class of all convex bodies is attained if $K$ is a triangle.

The minimum of $M_{p}(K)$ in the class of all centrally symmetric convex bodies is attained if $K$ is a parallelogram.

Proof. The second part of the statement easily follows from Theorem 4. Indeed, for a centrally symmetric convex body, the function $G_{p}(K-x)$ attains its maximum at the center of symmetry.

For a general convex figure $K$, Theorem 4 implies that

$$
M_{p}(K) \geq G_{p}\left(K-c_{K}\right) \geq G_{p}\left(T-c_{T}\right)=M_{p}(T),
$$

where $T$ is an equilateral triangle and the last equality follows from the symmetry of $T$.

\section{REFERENCES}

[A1] A. D. Aleksandrov, General method for majorizing the solutions of the Dirichlet problem, Sib. Math. Z. 8 (1966), 394-403. MR0217421 (36:511)

[A2] A. D. Aleksandrov, On mean values of support functions, Soviet Math. Dokl. 8 (1967), 149-153. MR0212681(35:3548)

[BM] J. Bourgain and V. Milman, New volume ratio properties for convex symmetric bodies in $\mathbb{R}^{n}$, Invent. Math. 88 (1987), 319-340. MR0880954 (88f:52013)

[BZ] Yu. D. Burago, V. A. Zalgaller, Geometric Inequalities, Springer-Verlag, Berlin, Heidelberg, 1988. MR0936419 (89b:52020)

[CCG] S. Campi, A. Colesanti and P. Gronchi, A note on Sylvester's problem for random polytopes in a convex body, Rend. Ist. Mat. Univ. Trieste 31 (1999), 79-94. MR.1763244 (2001d:52014)

[CG1] S. Campi and P. Gronchi, The $L^{p}$-Busemann-Petty centroid inequality, Adv. Math. 167 (2002), 128-141. MR1901248 (2003e:52011)

[CG2] S. Campi, P. Gronchi, On the reverse $L^{p}$-Busemann-Petty centroid inequality, Mathematika 49 (2002), 1-11. MR2059037 (2005d:52006)

[G1] R. J. Gardner, Geometric Tomography, Cambridge University Press, Cambridge, 1995. MR1356221 (96j:52006)

[G2] R. J. Gardner, The Brunn-Minkowski inequality, Bull. Amer. Math. Soc. 39 (2002), 355405. MR 1898210 (2003f:26035)

[GMR] Y. Gordon, M. Meyer and S. Reisner, Zonoids with minimal volume-product $-A$ new proof, Proc. AMS 104 (1988), 273-276. MR0958082 (89i:52015)

[L] E. Lutwak, Selected affine isoperimetric inequalities, Handbook of Convex Geometry (P. M. Gruber and J. M. Wills, eds.), North-Holland, Amsterdam, 1993, pp. 151-176. MR 1242979 (94h:52014)

[LYZ] E. Lutwak, D. Yang and G. Zhang, $L_{p}$ affine isoperimetric inequalities, J. Differential Geom. 56 (2000), 111-132. MR1863023 (2002h:52011)

[LZ] E. Lutwak and G. Zhang, Blaschke-Santaló inequalities, J. Differential Geom. 47 (1997), 1-16. MR.1601426 (2000c:52011)

[Ma1] K. Mahler, Ein Ubertragungsprinzip für konvexe Körper, Casopis Pêst. Mat. Fys. 68 (1939), 93-102. MR0001242(1:202c)

[Ma2] K. Mahler, Ein Minimalproblem für konvexe Polygone, Mathematica (Zutphen) B 7 (1939), 118-127.

[Me] M. Meyer, Convex bodies with minimal volume product in $\mathbb{R}^{2}$, Monatsh. Math. 112 (1991), 297-301. MR:1141097 (92k:52015)

[MP] M. Meyer and A. Pajor, On the Blaschke-Santaló inequality, Arch. Math. 55 (1990), 82-93. MR1059519 (92b:52013)

[Re1] S. Reisner, Random polytopes and the volume product of symmetric convex bodies, Math. Scand. 57 (1985), 386-392. MR0832364 (87g:52011)

[Re2] S. Reisner, Zonoids with minimal volume product, Math. Z. 192 (1986), 339-346. MR0845207 (87g:52022) 
[RS] C. A. Rogers and G. C. Shephard, Some extremal problems for convex bodies, Mathematika 5 (1958), 93-102. MR0104203 (21:2960)

[SR] Saint Raymond, Sur le volume des corps convexes symétriques, Sem. d'Initiation à l'Analyse 11 (1980-1981). MR0670798 (84j:46033)

[Sc] R. Schneider, Convex bodies: the Brunn-Minkowski theory, Cambridge University Press, Cambridge, 1993. MR1216521 (94d:52007)

[Sh] G. C. Shephard, Shadow systems of convex bodies, Israel J. Math. 2 (1964), 229-36. MR0179686 (31:3931)

[T] G. Talenti, Some estimates of solutions to Monge-Ampére type equations in dimension two, Ann. Sc. Norm. Super. Pisa IV (1981), 183-230. MR0623935(82h:53077)

Dipartimento di Matematica Pura e Applicata "G. Vitali", Università degli Studi di Modena e Reggio Emilia, Via Campi 213/B, 41100 Modena, Italy

E-mail address: Campi@unimo.it

Current address: Dipartimento di Ingegneria dell'Informazione, Università degli Studi di Siena, Via Roma 56, 53100 Siena, Italy

E-mail address: Campi@dii.unisi.it

Istituto per le Applicazioni del Calcolo - Sezione di Firenze, Consiglio Nazionale delle Ricerche Via Madonna del Piano, Edificio F, 50019 Sesto Fiorentino (Fi), Italy

E-mail address: P.Gronchi@fi.iac.cnr.it

Current address: Dipartimento di Matematica e Applicazioni per l'Architettura, Università degli Studi di Firenze, Piazza Ghiberti 27, 50122 Firenze, Italy

E-mail address: P.Gronchi@fi.iac.cnr.it 